\title{
On Weyl modules for the symplectic group
}

\author{
Ilaria Cardinali Antonio Pasini
}

\begin{abstract}
A rich information can be found in the literature on Weyl modules for $\operatorname{Sp}(2 n, \mathbb{F})$, but the most important contributions to this topic mainly enlighten the algebraic side of the matter. In this paper we try a more geometric approach. In particular, our approach enables us to obtain a sufficient condition for a module as above to be uniserial and a geometric description of its composition series when our condition is satisfied. Our result can be applied to a number of cases. For instance, it implies that the module hosting the Grassmann embedding of the dual polar space associated to $\operatorname{Sp}(2 n, \mathbb{F})$ is uniserial.
\end{abstract}

Keywords : symplectic groups, symplectic grasmmannians, Weyl modules.

MSC 2000: 20G05, 51A45, 51A50, 20E42, 20F40.

\section{Introduction}

Let $V$ be a $2 n$-dimensional vector space over a field $\mathbb{F}$ and, for a given nondegenerate alternating form $\alpha(.,$.$) of V$, let $G \cong \operatorname{Sp}(2 n, \mathbb{F})$ be the symplectic group associated with it and $\Delta$ the building associated with $G$. The elements of $\Delta$ of type $k=1,2, \ldots, n$ are the $k$-dimensional subspaces of $V$ totally isotropic for the form $\alpha$.

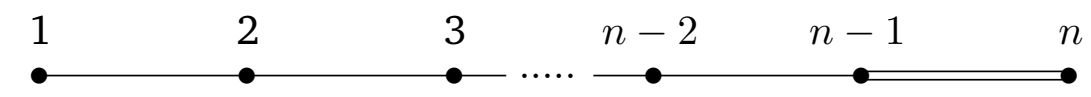

For $1 \leq k \leq n$, let $\mathcal{G}_{k}$ be the $k$-grassmannian of $\mathrm{PG}(V)$, where the $k$-subspaces of $V$ are taken as points. We recall that the lines of $\mathcal{G}_{k}$ are the sets

$$
l_{X, Y}:=\{Z \mid X \subset Z \subset Y, \operatorname{dim}(Z)=k\}
$$






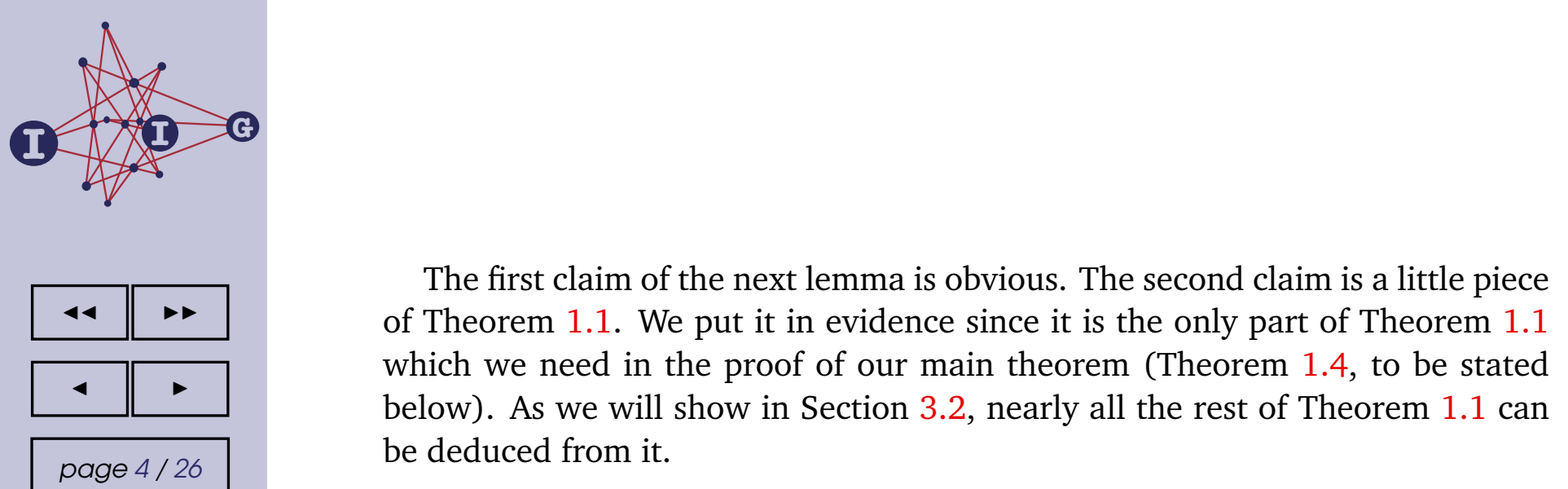

Lemma 1.3. (1) A nonnegative integer $r<k$ belongs to $J_{p}(k, n)$ if and only if $0 \in J_{p}(k-r, n-r)$, namely $k-r$ is even and $(k-r) / 2 \leq_{p} n-r+1$.

(2) The module $V_{k}$ admits a 1-dimensional section if and only if $0 \in J_{p}(k, n)$.

Clearly, if $S\left(V_{k}\right)=P_{k}$ then $P_{k}$ is a 1-dimensional section of $V_{k}$. In fact, it is the unique 1-dimensional section of $V_{k}$ since, according to Theorem 1.1, no two 1-dimensional sections can occur in the same composition series of $V_{k}$. On the other hand, it can happen that $V_{k}$ admits a 1-dimensional section but $P_{k} \not \subset V_{k}$ (see Remark 5.3).

We slightly change our notation by writing $W_{k, n}, V_{k, n}, \iota_{k, n}, \varepsilon_{k, n}$ and $P_{k, n}$ instead of $W_{k}, V_{k}, \iota_{k}, \varepsilon_{k}$ and $P_{k}$, in order to keep a record of the rank $n$ of $G$ and $\Delta$ in these symbols, but we refrain from extending this heavier notation further, thus keeping the symbols $G, \Delta, \mathcal{G}_{k}$ and $\Delta_{k}$ with no change.

In [8] we proved that, for any given value of the difference $h=n-k$, denoted by $n(h, p)$ the smallest $n$ for which $V_{k, n}$ is reducible, if $n=n(h, p)$ then $R\left(V_{k, n}\right)=P_{k, n}$ while if $n>n(h, p)$ then $R\left(V_{k, n}\right)$ contains a submodule spanned by 'local poles'. We shall explain in a few lines what local poles are.

Given a positive integer $r<k$ with $k-r$ even, for every $r$-element $X$ of $\Delta$ let $\mathcal{G}_{k, X}$ be the set of $k$-subspaces of $V$ that contain $X$ and $W_{k, n}^{X}$ the subspace of $W_{k, n}$ spanned by $\iota_{k, n}\left(\mathcal{G}_{k, X}\right)$. Also, let $\Delta_{k, X}$ be the set of $k$-elements of $\Delta$ that contain $X$ and $V_{k, n}^{X}$ the subspace of $V_{k, n}$ spanned by $\varepsilon_{k, n}\left(\Delta_{k, X}\right)$. Let $G_{X}$ be the stabilizer of $X$ in $G$ and let $K_{X}$ be the element-wise stabilizer of $\Delta_{k, X}$. Then $G_{X} / K_{X} \cong \operatorname{Sp}(2 n-2 r, \mathbb{F})$ and $K_{X}$ also fixes all elements of $\mathcal{G}_{k, X}$. Thus $G_{X} / K_{X}$ also acts on $W_{k, n}^{X}$. Moreover $W_{k, n}^{X} \cong W_{k-r, n-r}$ and $V_{k, n}^{X} \cong V_{k-r, n-r}$ as $\operatorname{Sp}(2 n-2 r, \mathbb{F})$-modules (see also Proposition 2.1 of this paper). As $k-r$ is even, $W_{k-r, n-r}$ admits a pole $P_{k-r, n-r}$. Let $P_{X}$ be the point of $\operatorname{PG}\left(W_{k, n}^{X}\right)$ corresponding to $P_{k-r, n-r}$ in the isomorphism $W_{k, n}^{X} \cong W_{k-r, n-r}$. Then $P_{X}$ is the unique fixed point of $G_{X}$ in its action on $\operatorname{PG}\left(W_{k, n}^{X}\right)$. We call $P_{X}$ the pole of $G_{X}$ in $W_{k, n}^{X}$, also the local pole of $G$ at $X$.

Suppose that $P_{k-r, n-r} \subset V_{k-r, n-r}$, namely $\operatorname{dim}\left(S\left(V_{k-r, n-r}\right)\right)=1$. Then $P_{X} \subset V_{k, n}^{X}$ and we can consider the following subspace of $V_{k, n}$ :

$$
\left.\mathcal{P}_{k, n}^{r}:=\left\langle P_{X}\right| X \text { is an } r \text {-element of } \Delta\right\rangle .
$$

If $P_{k, n} \subset V_{k, n}$ we put $\mathcal{P}_{k, n}^{0}:=P_{k, n}$. Let

$$
\widetilde{J}_{p}(k, n):=\left\{r \mid 0 \leq r<k, \operatorname{dim}\left(S\left(V_{k-r, n-r}\right)\right)=1\right\} .
$$







\subsection{Radical and 1-dimensional sections of $V_{k, n}$}

In the introduction, the radical $R\left(V_{k, n}\right)$ of $V_{k, n}$ has been defined as the largest proper submodule of $V_{k, n}$. We have also mentioned that $R\left(V_{k, n}\right)$ can be characterized as the intersection of all hyperplanes of $V_{k, n}$ spanned by $\varepsilon_{k, n}$-images of singular hyperplanes of $\Delta_{k}$ (Blok [5]). This characterization can be rephrased in the following way, more suited to our needs in this paper.

A non-degenerate bilinear form $\alpha_{k}(.,$.$) can be defined on W_{k, n}$ such that, for any two points $X$ and $Y$ of $\Delta_{k}$ and any non-zero vectors $x \in \varepsilon_{k, n}(X), y \in$ $\varepsilon_{k, n}(Y)$, we have $\alpha_{k}(x, y)=0$ if and only if $X$ and $Y$ are non-opposite as elements of $\Delta$ (see [8, Section 2]). Blok's characterization of $R\left(V_{k, n}\right)$ amounts to say that $R\left(V_{k, n}\right)=V_{k, n} \cap V_{k, n}^{\perp_{k}}$, where $\perp_{k}$ stands for the orthogonality relation with respect to $\alpha_{k}$.

We shall now describe when 1-dimensional sections occur. To this end, we consider pairs $(k, n)$ with a fixed difference $h=n-k$. It turns out that the decomposition of $V_{k, n}$ largely depends on this difference $h$. Let $N(h, p)$ be the smallest integer $n>h$ such that $p$ divides $\left(\begin{array}{c}1+\lfloor(n+h) / 2\rfloor \\ h+1\end{array}\right)$. The following proposition is a corollary of the proof of Theorem 1.1 by Premet and Suprunenko [15]. A different, more geometric proof of this proposition is given in [8, Section 5], but only valid when $p-1$ does not divide $h$. Another proof is given by De Bruyn [11].

Proposition 2.2. Let $h=n-k$. If $n<N(h, p)$ then $R\left(V_{k, n}\right)=0$. If $n=N(h, p)$ then $R\left(V_{k, n}\right)$ is 1-dimensional. If $n>N(h, p)$ then $\operatorname{dim}\left(R\left(V_{k, n}\right)\right)>1$.

In view of the next formula we need to state a few conventions. Let $h=$ $\sum_{j=0}^{\infty} h_{j} p^{j}$ be the expansion of $h$ to the base $p$. Let $e$ the smallest $j$ such that $h_{j}<p-1$. So,

$$
h=\left[(p-1) \cdot \sum_{j=0}^{e-1} p^{j}\right]+h_{e} p^{e}+h_{e+1} p^{e+1}+\cdots
$$

with $0 \leq h_{e}<p-1$. Note that $e=0$ is allowed in the above. In this case $h_{0}<p-1$. As remarked in [8, Section 5],

$$
N(h, p)=2\left(p-1-h_{e}\right) p^{e}+h .
$$

By claim (2) of Lemma 1.3, $V_{k, n}$ admits a 1-dimensional section if and only if $0 \in J_{p}(k, n)$, namely $k / 2 \leq_{p} n+1$.

Lemma 2.3. Let $k$ be even. Then $k / 2 \leq_{p} n+1$ if and only if $p^{e}$ divides $k$ and

$$
\frac{k}{2 p^{e}}=p^{t+1}-1-\sum_{j=0}^{t} h_{j+e} p^{j}+\sum_{i=1}^{r}\left[p^{s_{i}+t_{i}+1}-\sum_{j=s_{i}}^{s_{i}+t_{i}} h_{j+e} p^{j}\right]
$$


for an integer $t \geq-1$, a nonnegative integer $r$, positive integers $s_{1}, s_{2}, \ldots, s_{r}$ and nonnegative integers $t_{1}, t_{2}, \ldots, t_{r}$ such that

$$
\begin{aligned}
& t+r \geq 0, \quad t+1<s_{1}, \\
& s_{1}+t_{1}+1<s_{2}, \quad s_{2}+t_{2}+1<s_{3}, \ldots, s_{r-1}+t_{r-1}+1<s_{r} \text {, } \\
& p-1>h_{j+e} \quad \text { for } j=0,1, \ldots, t \text {, } \\
& h_{e+s_{i}} \neq 0 \quad \text { for } i=1,2, \ldots, r \text {, and } \\
& p-1>h_{e+s_{i}+j} \text { for } i=1,2, \ldots, r, j=1,2, \ldots, t_{i} \text {. }
\end{aligned}
$$

full screen

close

We will turn to the proof of this lemma in a few lines. We make a few remarks first. If $t=-1$ then $\sum_{j=0}^{t} h_{j+e} p^{j}=0$ by convention, while if $r=0$ then $\sum_{i=1}^{r}\left[p^{s_{i}+t_{i}+1}-\sum_{j=s_{i}}^{s_{i}+t_{i}} h_{j+e} p^{j}\right]=0$. Note that either $t \geq 0$ or $r>0$, since $t+r \geq 0$. Note also that, if we put $t=r=0$ in (3), then we obtain $n=N(h, p)$ as in (2).

Proof of Lemma 2.3. We only give a sketch of the proof of the 'only if' part of the lemma, leaving the rest and all details for the reader. Note that $n=k+h$. Let $k / 2=k_{0}+k_{1} p+k_{2} p^{2}+\cdots$ be the expansion of $k / 2$ to the base $p$. So,

$$
n+1=\left[2 k_{0}+2 k_{1} p+\cdots\right]+\left[\left(1+h_{e}\right) p^{e}+h_{e+1} p^{e+1}+h_{e+2} p^{e+2}+\cdots\right] .
$$

It follows that $k / 2 \leq_{p} n+1$ only if $k_{0}=k_{1}=\cdots=k_{e-1}=0$. Therefore, assuming that $k / 2 \leq_{p} n+1$, we obtain that $p^{e}$ divides $k / 2$. Assume first that $k_{e} \neq 0$ and let $t$ be the largest integer such that $k_{j} \neq 0$ for every $j=e, e+1, \ldots, e+t$. Then condition $k / 2 \leq_{p} n+1$ implies that $k_{j}=p-h_{j}-1$ for every $j=e, e+1, \ldots, e+t$. So,

$$
\sum_{j=0}^{t} k_{j+e} p^{j+e}=p^{t+e+1}-p^{e}-\sum_{j=0}^{t} h_{j+e} p^{j+e} .
$$

If $k_{j}=0$ for every $j>e+t$ then $k / 2 p^{e}$ is as in (3) with $r=0$. Suppose that $k_{j} \neq$ 0 for some $j>t+e$ and let $s_{1}$ be the smallest integer $j>t$ such that $k_{j+e} \neq 0$. As $k_{e+t+1}=0$ by the choice of $t$, we have $s_{1}>t+1$. Moreover, $k / 2 \leq_{p} n+1$ forces $k_{e+s_{1}}=p-h_{e+s_{1}}$. Hence $h_{e+s_{1}} \neq 0$, because $k_{e+s_{1}} \neq 0$. Let $t_{1}$ be the largest integer such that $k_{j} \neq 0$ for every $j=e+s_{1}, e+s_{1}+1, \ldots, e+s_{1}+t_{1}$. Then $k_{e+s_{1}+j}=p-1-h_{e+s_{1}+j}$ for $j=1,2, \ldots, t_{1}$. For these values of $j$ we have $h_{e+s_{1}+j}<p-1$ because $k_{e+s_{1}+j} \neq 0$. Moreover,

$$
\sum_{j=s_{1}}^{s_{1}+t_{1}} k_{j+e} p^{j+e}=p^{t_{1}+s_{1}+e+1}-\sum_{j=s_{1}}^{s_{1}+t_{1}} h_{j+e} p^{j+e} .
$$

It is now clear how to go on. We end up with (3). We have assumed $k_{e} \neq 0$. If $k_{e}=0$ then we still obtain (3) but with $t=-1$. In this case $s_{1}$ is the smallest integer $j$ such that $k_{j+e} \neq 0$. 
Proposition 2.4. We have $0 \in J_{p}(k, n)$ if and only if $k$ is even, $p^{e}$ divides $k$ and $k / 2 p^{e}$ is as in (3) of Lemma 2.3.

Proof. By claim (2) of Lemma 1.3, $0 \in J_{p}(k, n)$ if and only if $k$ is even and $k / 2 \leq_{p} n+1$. Lemma 2.3 yields the conclusion.

\subsection{The basic series and the pole}

full screen

close

quit

For $0 \leq i \leq\lfloor k / 2\rfloor$, where $\lfloor k / 2\rfloor$ is the integral part of $k / 2$, we denote by $V_{k-2 i}^{(k, n)}$ the subspace of $W_{k, n}$ spanned by the vectors $\iota_{k, n}(X)$ for a $k$-subspace $X$ of $V$ with $\operatorname{dim}\left(X \cap X^{\perp}\right) \geq k-2 i$. In particular, $V_{k}^{(k, n)}=V_{k, n}$. Clearly, $V_{k-2 i}^{(k, n)}$ is $G$-invariant and $V_{k-2 i}^{(k, n)} \subseteq V_{k-2 j}^{(k, n)}$ for $0 \leq i \leq j \leq\lfloor k / 2\rfloor$. Note that $k-2\lfloor k / 2\rfloor$ is equal to 0 or 1 according to whether $k$ is even or odd. In any case, $V_{k-2\lfloor k / 2\rfloor}^{(k, n)}=$ $W_{k, n}$. We put $V_{k+2}^{(k, n)}:=0$, by convention. The series of the $G$-submodules of $W_{k, n}$ defined above is called the basic series of $G$ in $W_{k, n}$ :

$$
0=V_{k+2}^{(k, n)} \subseteq V_{k}^{(k, n)} \subseteq V_{k-2}^{(k, n)} \subseteq \cdots \subseteq V_{k-2\lfloor k / 2\rfloor}^{(k, n)}=W_{k, n} .
$$

When $k$ is odd the clause $i<k / 2-1$ is equivalent to $i<\lfloor k / 2\rfloor$. When $k$ is even and $i=k / 2-1$ then $V_{2}^{(k, n)}$ is a hyperplane of $V_{0}^{(k, n)}=W_{k, n}$.

Let $0 \leq i<k / 2$. Given a totally singular $(k-2 i)$-subspace $X$ of $V$, choose a $k$-subspace $Y$ of $V$ such that $Y \cap Y^{\perp}=X$ and put

$$
\varphi_{i}(X):=\iota_{k, n}(Y)+V_{k-2 i+2}^{(k, n)} \in \operatorname{PG}\left(V_{k-2 i}^{(k, n)} / V_{k-2 i+2}^{(k, n)}\right) .
$$

Proposition 2.5. The mapping $\varphi_{i}$ is well defined, it is an embedding of $\Delta_{k-2 i}$ in $\mathrm{PG}\left(V_{k-2 i}^{(k, n)} / V_{k-2 i+2}^{(k, n)}\right)$ and it is isomorphic to the natural embedding $\varepsilon_{k-2 i, n}$ of $\Delta_{k-2 i}$.

(See [8, Theorem 3.5]; we warn that the universality of $\varepsilon_{k-2 i, n}$ is exploited in the proof of that theorem.) As recalled in the introduction of this paper, if $k$ is odd then $G$ acts fixed-point-freely on $\mathrm{PG}\left(W_{k, n}\right)$ while when $k$ is even $G$ fixes exactly one point $P_{k, n}$ of $\mathrm{PG}\left(W_{k, n}\right)$, called the pole of $G$ in $W_{k, n}$. Clearly, $G$ stabilizes the 1-dimensional subspace $\left(V_{2}^{(k, n)}\right)^{\perp_{k}}$ of $W_{k, n}$ (where $\perp_{k}$ is defined as in Section 2.3). Hence $P_{k, n}=\left(V_{2}^{(k, n)}\right)^{\perp_{k}}$.

Proposition 2.6. $P_{k, n}=\left\langle v_{P_{k, n}}\right\rangle$ where $v_{P_{k, n}}=\sum_{J \in\left(\begin{array}{c}I \\ k / 2\end{array}\right)} e_{J} \wedge f_{J}$.

(See [8, Lemma 4.2].) We take the vector $v_{P_{k, n}}$ as the canonical representative of $P_{k, n}$. The following is also proved in [8, Theorem 4.3]: 
Proof. Let $\Theta_{k}$ be the set of ordered triples $(X, Y, Z)$ of pairwise disjoint (and possibly empty) subsets of $I=\{1,2, \ldots, n\}$ such that $|X|+2|Y|+|Z|=k$. Every vector $v \in W_{k, n}$ can be written in a unique way as a linear combination

$$
v=\sum_{(X, Y, Z) \in \Theta_{k}} \lambda_{X, Y, Z} e_{X} \wedge\left(e_{Y} \wedge f_{Y}\right) \wedge f_{Z} .
$$

go back

full screen

close

quit
ACADEMIA PRESS

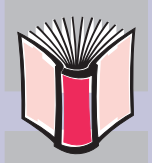

$\widehat{\underline{\text { IIIIII }}}$ UNIVERSITEIT GENT

Suppose that $U(v)=v$. This condition is equivalent to $L(U)(v)=0$, where $L(U)$ is the nilpotent subalgebra of the Lie algebra $L(G)$ of $G$ corresponding to $U$. Considering elements of $L(U)$ corresponding to long roots, it is straightforward to check that $\lambda_{X, Y, Z}=0$ whenever $Z \neq \emptyset$. So,

$$
v=\sum\left(\lambda_{X, Y} e_{X} \wedge\left(e_{Y} \wedge f_{Y}\right)|X \cap Y=\emptyset,| X|+2| Y \mid=k\right)
$$

where $\lambda_{X, Y}:=\lambda_{X, Y, \emptyset}$. We can now consider elements of $L(U)$ corresponding to short simple roots or sums of short simple roots. Given two disjoint subsets $X, Y \subset I$ such that $|X|+2|Y|=k$, we write $X<Y$ if every element of $X$ is smaller than all elements of $Y$. Recalling that the elements of $L(U)$ map $v$ to 0 , one can see that $\lambda_{X, Y}=0$ only if $X<Y$ and that if $X<Y, Y^{\prime}$ then $\lambda_{X, Y}=\lambda_{X, Y^{\prime}}$. We leave details for the reader. At this stage,

$$
v=\sum\left(\lambda_{X, Y} e_{X} \wedge\left(e_{Y} \wedge f_{Y}\right)|X<Y,| X|+2| Y \mid=k\right) .
$$

It remains to prove that $X$ is an initial segment of $I$. This can be seen by considering elements of $L(U)$ corresponding to sums of short simple roots and one long root. Again, we leave details for the reader. The first claim of the lemma is proved.

Turning to the second claim, note that if $B(\langle v\rangle)=\langle v\rangle$ then $U(v)=v$. Therefore, if $\langle v\rangle$ is fixed by $B$, then $v \in\left\langle\widehat{e}_{i} \wedge \widehat{v}_{i}\right\rangle_{i=0}^{\lfloor k / 2\rfloor}$, say $v=\sum_{i=0}^{\lfloor k / 2\rfloor} \lambda_{i} \widehat{e}_{i} \wedge \widehat{v}_{i}$. Let now $H$ be the Cartan subgroup of $B$ stabilizing each of the subspaces $\left\langle\widehat{e}_{i} \wedge \widehat{v}_{i}\right\rangle$. Recall that $H \cong\left(\mathbb{F}^{*}\right)^{n}$. If $g \in H$ corresponds to $\left(t_{1}, \ldots, t_{n}\right) \in\left(\mathbb{F}^{*}\right)^{n}$, then $g$ maps $v$ to

$$
g(v)=\sum_{i=0}^{\lfloor k / 2\rfloor}\left(t_{1} \cdots t_{k-2 i}\right) \cdot \lambda_{i} \widehat{e}_{i} \wedge \widehat{v}_{i}
$$

The vectors $\widehat{e}_{i} \wedge \widehat{v}_{i}$ are independent, the scalars $t_{1}, \ldots, t_{n}$ are arbitrary elements of $\mathbb{F}^{*}$ and $\mathbb{F}^{*}$ contains at least two elements. It follows that $H(v) \in\langle v\rangle$ if and only if $v$ is proportional to one of the vectors $\widehat{e}_{i} \wedge \widehat{v}_{i}$.

Lemma 3.2. For $J \subset I=\{1,2, \ldots, n\}$, let $j=|J|$, put $V^{J}=\left\langle e_{i}, f_{i}\right\rangle_{i \in I \backslash J} \cong$ $V(2 n-2 j, \mathbb{F})$ and $W_{k-j}^{J}=\wedge^{k-j} V^{J} \cong W_{k-j, n-j}$ and let $V_{k-j}^{J} \cong V_{k-j, n-j}$ be the subspace of $W_{k-j}^{J}$ spanned by the vectors representing totally isotropic subspaces of $V^{J}$. Then $e_{J} \wedge V_{k-j}^{J}=\left(e_{J} \wedge W_{k-j}^{J}\right) \cap V_{k, n}$. 





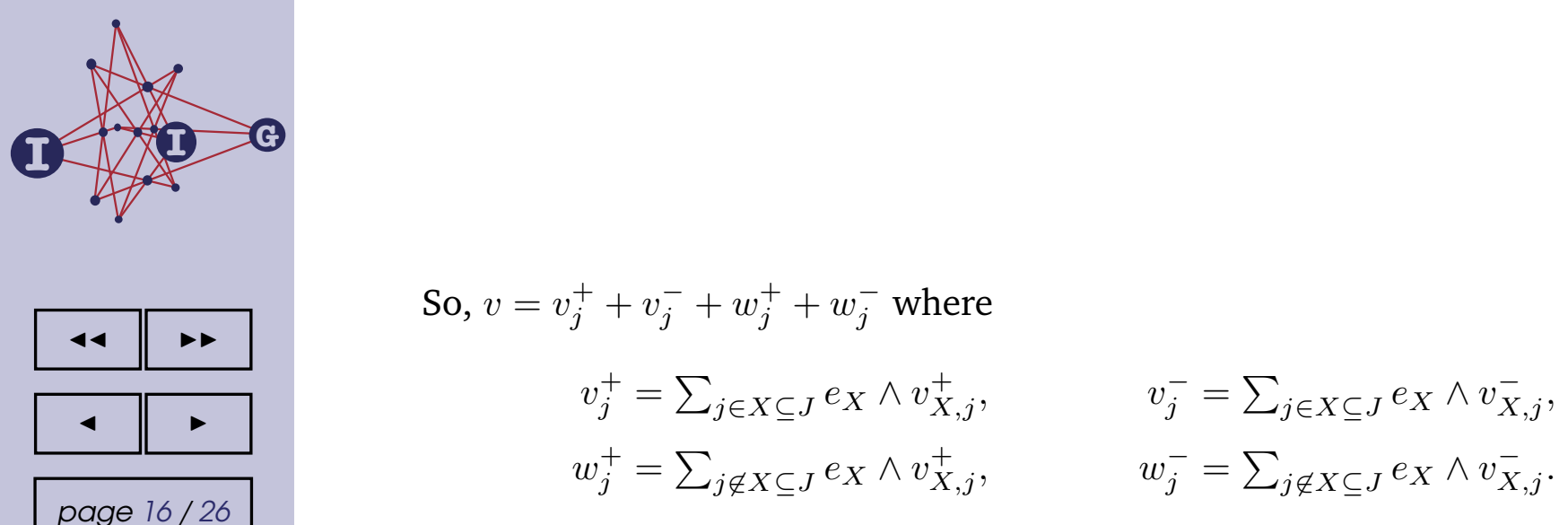

Let $b$ be the element of $B$ fixing $e_{i}$ and $f_{i}$ for $i \neq j$ and sending $e_{j}$ to $t e_{j}$ and $f_{j}$ to $t^{-1} f_{j}$. Then

$$
b(v)-t v \in S
$$

Indeed $b\left(e_{J}\right)=t e_{J}$ in $V_{r, n}$ because $j \in J$ and $b(v+S)=t(v+S)$. (Recall that $B$ acts on $v+S$ as on $e_{J}$.) On the other hand,

$$
b(v)=v_{j}^{+}+t v_{j}^{-}+t^{-1} w_{j}^{+}+w_{j}^{-} .
$$

By substituting (5) in (4) we obtain that $(1-t) v_{j}^{+}+\left(t^{-1}-t\right) w_{j}^{+}+(1-t) w_{j}^{-} \in S$. If $t \neq 1$ then

$$
v_{j}^{+}+\frac{t^{-1}-t}{1-t} w_{j}^{+}+w_{j}^{-} \in S
$$

By putting $t=-1$ in (6) we obtain that

$$
v_{j}^{+}+w_{j}^{-} \in S
$$

Suppose that $\mathbb{F}$ contains at least four elements. Then we can assume to have chosen $t \neq 1,-1$ in (6). Hence $\left(t^{-1}-t\right) /(1-t) \neq 1$ and by comparing (6) with (7) we also obtain that $w_{j}^{+} \in S$. Therefore $v_{j}^{+}+w_{j}^{+}+w_{j}^{-} \in S$ and we can assume to have chosen the representative $v$ of $v+S$ in such a way that $v=v_{j}^{-}=\sum_{j \in X \subseteq J} e_{X} \wedge v_{X, j}^{-}$. However this holds for every $j \in J$. By considering the elements of $J$ in some order and adjusting at every step the choice of $v$ as explained above, we can eventually assume to have chosen $v$ so that $v=e_{J} \wedge v_{J}$ where $v_{J} \in W_{k-r}^{J}=\wedge^{k-r}\left\langle e_{r+1}, \ldots, e_{n}, f_{r+1}, \ldots, f_{n}\right\rangle$ (notation as in Lemma 3.2). On the other hand, $e_{J} \wedge v_{J} \in S^{\prime} \subseteq V_{k, n}$. By Lemma 3.2, $v_{J} \in V_{k-r}^{J}$. So, $\widehat{f}\left(e_{J}\right)=e_{J} \wedge v_{J}+S$ with $v_{J} \in V_{k-r}^{J}$. Note that no use is made in the above argument of the hypothesis that $S^{\prime} / S$ is irreducible. We have only exploited the existence of a surjective homomorphism of $G$-modules $\widehat{f}: V_{r, n} \rightarrow S^{\prime} / S$.

Let now $\mathbb{F}=\mathbb{F}_{3}$. (Recall that $\mathbb{F} \neq \mathbb{F}_{2}$ because char $(\mathbb{F}) \neq 2$ by assumption.) Then (6) and (7) only allow us to choose $v=\sum_{X \subset J} e_{X} \wedge f_{J \backslash X} \wedge v_{X}$ with $v_{X} \in$ $W_{k-r}^{J}$ for every $X \subseteq J$. However, we can get out from this blind alley by the following trick. Note first that the $G$-invariant subspaces of $V_{k, n}$ are precisely the $L(G)$-invariant subspaces of $V_{k, n}$, where $L(G)$ is the Lie algebra of $G$. However, $L(G)$ bears the structure of a vector space. So, given any extension $\overline{\mathbb{F}}$ of $\mathbb{F}$ (for instance, $\overline{\mathbb{F}}=\mathbb{F}_{9}$ ), we can consider the scalar extensions $\bar{L}(G):=\overline{\mathbb{F}} \otimes L(G)$ as 


Suppose that $r \in \widetilde{J}_{p}(k, n)$, namely the socle $S\left(V_{k-r, n-r}\right)$ of $V_{k-r, n-r}$ is 1-dimensional. So, by Lemma $1.2, k-r$ is even and, for every $r$-element $X$ of $\Delta$, the local pole $P_{X}$ of $G$ at $X$ is equal to $S\left(V_{k, n}^{X}\right)$. (Recall that $P_{X}$ is the unique point of $\mathrm{PG}\left(W_{k, n}^{X}\right)$ fixed by the stabilizer $G_{X}$ of $X$ in $G$; see Section 1.) Then $n-r \geq N(h, p)$ by Proposition 2.2 applied to $V_{k-r, n-r}$. Hence $n>N(h, p)$. Therefore $\operatorname{dim}\left(R\left(V_{k, n}\right)\right)>1$ again by Proposition 2.2, but now applied to $V_{k, n}$.

As in Section 1, let $\mathcal{P}_{k, n}^{r}$ be the subspace of $V_{k, n}$ spanned by the 1-dimensional subspaces $P_{X}$, for $X$ an $r$-element of $\Delta$. Clearly, $\mathcal{P}_{k, n}^{r}$ is stabilized by $G$. We call $\mathcal{P}_{k, n}^{r}$ a geometric submodule of $V_{k, n}$, also geometric submodule of type $r$. Define the following map

$$
\begin{aligned}
\pi_{k, n}^{r}: \Delta_{r} & \rightarrow \operatorname{PG}\left(\mathcal{P}_{k, n}^{r}\right), \\
X & \mapsto P_{X} .
\end{aligned}
$$

Theorem 4.1. The mapping $\pi_{k, n}^{r}$ is an embedding of $\Delta_{r}$. Moreover $\pi_{k, n}^{r}$ is G-homogeneous and there exists a morphism from the natural embedding $\varepsilon_{r, n}$ of $\Delta_{r}$ to $\pi_{k, n}^{r}$.

Proof. Assume $r<n$, to fix ideas. The case of $r=n$ can be dealt with in a similar way, modulo minor modifications, which we leave to the reader.

We first show that lines of $\Delta_{r}$ are mapped onto lines of $\mathcal{P}_{k, n}^{r}$. Let $X_{1}$ and $X_{2}$ be two distinct collinear points of $\Delta_{r}$. They are $r$-dimensional totally isotropic subspaces of $V$. As they are assumed to be collinear, without loss of generality we may suppose that $X_{1}=\left\langle e_{1}, \ldots, e_{r-1}, e_{r}\right\rangle$ and $X_{2}=\left\langle e_{1}, \ldots, e_{r-1}, e_{r+1}\right\rangle$. So, a point $X_{3} \neq X_{1}, X_{2}$ on the line of $\Delta_{r}$ through $X_{1}$ and $X_{2}$ corresponds to an $r$-dimensional totally isotropic subspace of the form $\left\langle e_{1}, \ldots, e_{r-1}, e_{r}+t e_{r+1}\right\rangle$, $t \in \mathbb{F} \backslash\{0\}$.

By Proposition 2.6 and the proof of Proposition 2.1, we obtain that $P_{X_{1}}=$ $\left\langle v_{1}\right\rangle$ and $P_{X_{2}}=\left\langle v_{2}\right\rangle$, where

$$
\begin{aligned}
& v_{1}=\sum_{J \in\left(\begin{array}{c}
\{r+1, r+2, \ldots, n\} \\
(k-r) / 2
\end{array}\right)} e_{J} \wedge f_{J} \wedge\left(e_{1} \wedge \cdots \wedge e_{r-1} \wedge e_{r}\right), \\
& v_{2}=\sum_{J \in\left(\begin{array}{c}
\{r, r+2, \ldots, n\} \\
(k-r) / 2
\end{array}\right)} e_{J} \wedge f_{J} \wedge\left(e_{1} \wedge \cdots \wedge e_{r-1} \wedge e_{r+1}\right) .
\end{aligned}
$$

In order to compute $P_{X_{3}}$ we need to extend the basis $\left\{e_{1}, \ldots, e_{r-1}, e_{r}+t e_{r+1}\right\}$ of $X_{3}$ to a basis of $X_{3}^{\perp}$ by adding a hyperbolic basis $B$ of a complement of $X_{3}$ in $X_{3}^{\perp}$. We make the following choice:

$$
B=\left\{e_{r+2}, e_{r+3}, \ldots, e_{n}, \frac{1}{t} f_{r+1}-f_{r}, f_{r+2}, f_{r+3}, \ldots, f_{n}, e_{r}\right\}
$$







$$
p \text { divides }\left(\begin{array}{l}
n \\
x
\end{array}\right) \text { iff }|x|_{p} \geq p-1-h .
$$

It follows that $p$ divides $\left(\begin{array}{c}n \\ k / 2\end{array}\right)$. Indeed $k / 2=p^{t}-1-h=p^{t}-p+[p-1-h]$.

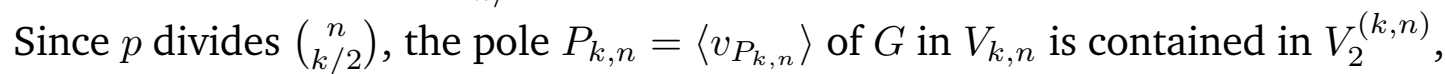
by Proposition 2.7. Let $j<k / 2$ be the least nonnegative integer such that $v_{P_{k, n}} \in V_{k-2 j}^{(k, n)}$, namely $v_{P_{k, n}} \in V_{k-2 j}^{(k, n)} \backslash V_{k-2 j+2}^{(k, n)}$. In order to prove the lemma we only must show that $j=0$.

Let $r:=2(p-1-h)$. By (9), $r$ is the smallest even integer such that $p$ divides $\left(\begin{array}{c}n \\ r / 2\end{array}\right)$. Hence $k-2 j \geq r$ by the minimality of $r$. So, $j \leq(k-r) / 2=p^{t}-p$. In order to align our notation to that of Proposition 2.8, we set $k-2 j=r+2 i$. So, $0 \leq i \leq p^{t}-p$ and the equality $j=0$, which we want to prove, is equivalent to $i=p^{t}-p$.

By condition (1) of Proposition 2.8, $p$ divides $\left(\begin{array}{c}n \\ r / 2+i\end{array}\right)$. Hence $|r / 2+i|_{p} \geq$ $p-1-h$, by equivalence (9). As $r / 2=p-1-h$ and $h<p-1$, the previous inequality is equivalent to the following:

$$
|i|_{p} \leq h
$$

On the other hand, condition (2) of Proposition 2.8 implies that $r / 2+i \leq_{p} n+1$, namely

$$
p-1-h+i \leq_{p} 2\left(p^{t}-1\right)-h+1=2 p^{t}-1-h .
$$

In view of (10), condition (11) implies $|i|_{p}=0$ and $i \leq_{p} p^{t}-p$. So,

$$
i=i_{1} p+i_{2} p^{2}+\cdots+i_{t-1} p^{t-1}
$$

where $i_{1}, i_{2}, \ldots, i_{t-1} \in\{0, p-1\}$. Consider now the binomial coefficient

$$
\left(\begin{array}{c}
k / 2 \\
k / 2-j
\end{array}\right)=\left(\begin{array}{c}
k / 2 \\
r / 2+i
\end{array}\right)=\left(\begin{array}{c}
p^{t}-1-h \\
p-1-h+i
\end{array}\right)
$$

By exploiting formula (8) one can see that $p$ divides $\left(\begin{array}{c}k / 2 \\ r / 2+i\end{array}\right)$ if and only if

$$
p+i_{1} p+i_{2} p^{2}+\cdots+i_{s} p^{s}>p^{s+1}
$$

for at least one $s \in\{1, \ldots, t-1\}$. However, this is impossible, since $p+i_{1} p+$ $i_{2} p^{2}+\cdots+i_{s} p^{s} \leq p^{s+1}$. Therefore, $p$ does not divide $\left(\begin{array}{c}k / 2 \\ k / 2-j\end{array}\right)$. In view of Proposition 2.8 (3), the prime $p$ neither divides

$$
\left(\begin{array}{c}
n-k+2 j \\
j
\end{array}\right)=\left(\begin{array}{c}
n-r-2 i \\
(k-r) / 2-i
\end{array}\right)=\left(\begin{array}{c}
2\left(p^{t}-p\right)+h-2 i \\
p^{t}-p-i
\end{array}\right) .
$$



